\title{
Role of Antiphoso lipid anti-bodies and Antinuclear anti-bodies in pregnancy outcome and its treatment for better outcome
}

\author{
Alka Dani ${ }^{1}$, Abhishek $^{2 *}$ \\ ${ }^{1}$ Associate Professor, ${ }^{2}$ Assistant Professor, Department of OBGY, Dr. D.Y.Patil Medical College, Hospital and Research Centre, Nerul, Navi \\ Mumbai INDIA. \\ Email: alka.dani@gmail.com
}

$\underline{\text { Abstract }}$

\begin{abstract}
Background: Patients with raised titre of ANA antibodies and APLA during pregnancy have more guarded prognosis like recurrent pregnancy loss, preterm delivery, lUGR, oligohydramnios, stillbirth and maternal complications like severe rhehmatoid arthritis, SLE,ITP, rashes, multiorgan failure in cases of positive ANA test and severe preeclampsia, venous and arterial thrombosis, stroke, coronary artery thrombosis if APLA is positive. Aims and objectives: In this study our objectives are to assess the role of ANA and APLA on maternal and fetal health and to diagnose and treat the condition early in pregnancy to improve the outcomes. Material and Methods: In this study 55 cases with h/o RPL and adverse maternal and fetal outcome in previous pregnancies and subchorionic haemorrhage in first trimester during current pregnancy were studied for presence of ANA, LAC and ACLA and their titers and serial sonography was done. Patient's councelling and treatment started accordingly and test was repeated after 12 weeks and maternal and fetal outcome was noted and analysed. Result- Out of 55patients $63.6 \%$ were ANA+ positive and $29.1 \%$ were ANA2+ positive. ACLA was positive in $12.7 \%$ and LAC in $3.3 \%$ of patients. $38.2 \%$ of patients had PIH, $20 \%$ GDM, $20 \%$ hypothyroidism and $12.7 \%$ patients had hyperthyroidism. In $63.6 \%$ patients, USG findings were abnormal. $78.2 \%$ patients had good fetal outcome while $21=8 \%$ patients had poor fetal outcome. Conclusion: ANA and APLA test should not be done as a routine but only in risk cases for early detection and prompt treatment so as to improve pregnancy course and fetal outcome.
\end{abstract}

Keywords: Recurrent pregnancy loss, Antinuclear antibodies, Antiphospholipid antibodies.

*Address for Correspondence:

Dr Abhishek, Assistant Professor, Department of OBGY, Dr. D.Y.Patil Medical College, Hospital and Research Centre, Nerul, Navi Mumbai INDIA.

Email: alka.dani@gmail.com

Received Date: 02/12/2019 Revised Date: 08/01/2020 Accepted Date: 11/02/2020

DOI: https://doi.org/10.26611/10121331

\begin{tabular}{|l|l|}
\hline \multicolumn{2}{|c|}{ Access this article online } \\
\hline Quick Response Code: & Website: \\
\hline & www.medpulse.in \\
\cline { 2 - 2 } & \\
\hline
\end{tabular}

\section{INTRODUCTION}

Patients who have raisedtitre of antinuclear anti-bodies (ANA) and antiphosolipid anti-bodies(APLA) during pregnancy have a more guarded prognosis like recurrent miscarriage, pre-term delivery, intra-uterine growth retardation (IUGR), oligohydramnios, still birth and maternal complications like severe rheumatoid arthritis, SLE, auto immune thrombocytopenia, rashes and multiorgan failure in case of positive ANA test and severe preeclampsia, venous and arterial thrombosis, stroke, coronary artery thrombosis if APLA is positive. APLA includes lupus anticoagulant (LAC) $\mathrm{AB}$ and anticardolipin (ACL) AB. Incidence of LAC in India is $15-30 \%$ and ACL is $23-86 \%$. These patients are more likely to get benefitted by therapeutic interventions like Aspirin, Heparin and Prednisolone. The ANA Test was designed by Dr. George Friou in 1957. It can also refer to as fluorescent antinuclear anti-body test (FANA). It is a sensitive screening test used to detect autoimmune diseases. ANA can be found in approximately $5 \%$ of normal population in low titers. ANA titer of $<=1: 40$ are considered negative. Those with higher titers have been found to have a risk of RPL and are more

\footnotetext{
How to cite this article: Alka Dani, Abhishek. Role of Antiphoso lipid anti-bodies and Antinuclear anti-bodies in pregnancy outcome and its treatment for better outcome. MedPulse International Journal of Gynaecology. March 2020; 13(3): 75-80. http://medpulse.in/Gynacology/index.php
} 
likely to be benefitted from Prednisone. In 1983 Hughes first described patients with the combination of clinical features (i.e. thrombosis, thrombocytopenia, RPL, IUGR, pre-eclampsia) associated with the presence of APLA and LAC. Primary method of diagnosis requires clinical and laboratory findings with the clinical criteria and should be tested for both LAC and ACL immunoglobulins (IgG and IgM).

Patho-physiology:

Human APLA reacts with endothelial structures ${ }^{[1]}$ which disturbs the $\mathrm{PGE}_{2} /$ Thromboxane production balance ${ }^{2}$, interaction with platelet PLs with consequent upregulation of platelet aggregation ${ }^{[3]}$, dysregulation of compliment activation and ${ }^{[4]}$ interaction of APL with phosphatidyl serine expose during trophoblast syncytium formation resulting in more direct effect of APLA on placental structure.

APS is associated with increased maternal morbidity and mortality as well as increased perinatal MMR. It is associated with increased risk of venous and arterial thrombosis, coronary artery occlusion. There is increased incidence of pre-eclampsia usually before 34 weeks, premature deliveries, infertility, spontaneous abortion and still births. MOFD has been described during pregnancies by Asherson $^{[1]}$ and during puerperium by Kochenom. ${ }^{2}$ Neonatal morbidity and mortality is due to severe preeclampsia and IUGR. Rate of fetal loss can be reduced by $20 \%$ by therapy (Aspirin and Heparin) as described by Cowchok et $a l^{3}$.

Aims and Objectives

To assess the role of Antinuclear antibody and Antiphospholipid antibody on maternal and fetal health. To diagnose and treat the condition early in pregnancy to improve outcomes. To study relation of relative titers of ANA with other anti-bodies and with adverse pregnancy outcomes.

\section{MATERIAL AND METHODS}

This prospective case study was conducted in a tertiary hospital of Mumbai from February 2009 to April 2011. Ethical committee approval was taken. After taking informed written consent, 50 cases with history of RPL and adverse maternal and fetal outcome in previous pregnancies like pre-term birth, IUGR, miscarriage, still births, IUFD, severe PIH in second trimester, subchorionic haemorrhage in first trimester during current pregnancy were included in the study and those with history of MTP, chronic hypertension without pregnancy losses and with normal pregnancy course were excluded from the study. Routine anti-natal and special investigations (ANA, LAC, ACLA) were done along with serial sonography. After the test results patient's counselling was done for probability of adverse pregnancy course (PIH, GDM and Thyroid dysfunction) and increased risk of adverse fetal outcome (missed abortion, pre-term labour, oligohydramnios, IUFD, IUGR). Patients with positive ANA, ACLA, LAC were then referred to the hospital rheumatologist for treatment and other immunological disorders. Then tests were repeated after 12 weeks and patients were also treated for PIH, GDM and thyroid dysfunction if present. USG was done in first trimester to detect subchorionichaemorrhage, missed abortion, blighted ovum and for nuchal translucency along with double marker test to rule out chromosomal anomalies. Second trimester anomalies scan and triple marker test was done between 18-20 weeks. GCT was done between 24-26 weeks. GTT was done if GCT was abnormal to detect GDM. Weight and BP were checked during each antenatal visit. During third trimester, along with routine checkup colour doppler studies were done on USG every 3-4 weeks to check fetal growth. Patients were asked to keep strict daily fetal movements count. Low dose of Aspirin was given to treat patients with increased APLA and ANA titers. Prednisolone and Hydroxychloroquine were also given to patient with autoimmune disorders. Aspirin and Prednisolone were stopped at 36 weeks of gestation. All the information was filled in the predesigned Performa. Data analysis was done using software SPSS for windows v. 15.0

\section{RESULTS}

Table 1: Descriptive Statistics of Demographic Variables

\begin{tabular}{cccccc}
\hline Variables & N & Mean & Stdev & Median & IQR \\
\hline Age(yrs) & 55 & 29.6727 & 5.4128 & 29 & 8 \\
Married(yrs) & 55 & 4.4545 & 3.0901 & 4 & 5 \\
Gravida & 55 & 2.1818 & 1.4154 & 2 & 2 \\
Parity & 55 & 0.5636 & 0.7641 & 0 & 1 \\
Abortion & 55 & 0.6727 & 1.0193 & 0 & 1 \\
Live Births & 55 & 0.4000 & 0.7354 & 0 & 1 \\
\hline
\end{tabular}

Table 2: Distribution of study group as per history of Hypertension

\begin{tabular}{ccc}
\hline HTN & No. of Females & Percentage \\
\hline PIH & 21 & 38.2 \\
No & 34 & 61.8 \\
\hline Total & 55 & 100.0
\end{tabular}

Table 3: Distribution of study group as per history of Diabetes

\begin{tabular}{ccc}
\hline Diabetes MOD & No. of Females & Percentage \\
\hline GDM & 11 & 20.0 \\
No & 44 & 80.0 \\
\hline Total & 55 & 100.0 \\
\hline
\end{tabular}

Table 4: Distribution of study group as per Thyroid function test

\begin{tabular}{ccc}
\hline Thyroid function test & No. of Females & Percentage \\
\hline Hypothyroidism & 11 & 20.0 \\
Hyperthyroidism & 7 & 12.7 \\
No & 37 & 67.3 \\
\hline Total & 55 & 100.0
\end{tabular}


Table 5: Distribution of study group as per result of ANA

\begin{tabular}{ccc}
\hline ANA & No. of Females & Percentage \\
\hline $1+$ & 35 & 63.6 \\
$2+$ & 16 & 29.1 \\
No & 4 & 7.3 \\
\hline Total & 55 & 100.0 \\
\hline
\end{tabular}

Table 6: Distribution of study group as per result of $\mathrm{ACL}$

\begin{tabular}{ccc}
\hline ACL & No. of Females & Percentage \\
\hline Positive & 7 & 12.7 \\
Negative & 48 & 87.3 \\
\hline Total & 55 & 100.0 \\
\hline
\end{tabular}

Table 7: Distribution of study group as per result of $\mathrm{AL}$

\begin{tabular}{ccc}
\hline ACL & No. of Females & Percentage \\
\hline Positive & 2 & 3.6 \\
Negative & 53 & 96.4 \\
\hline Total & 55 & 100.0
\end{tabular}

Table No 8: Distribution of study group as per USG finding

\begin{tabular}{ccc}
\hline USG & No. of Females & Percentage \\
\hline Normal & 20 & 36.4 \\
Abnormal & 35 & 63.6 \\
\hline Total & 55 & 100.0 \\
\hline
\end{tabular}

Table No 9: Distribution of study group as per history of Treatment taken

\begin{tabular}{ccc}
\hline Treatment taken & No. of Females & Percentage \\
\hline Yes & 39 & 70.9 \\
No & 16 & 29.1 \\
\hline Total & 55 & 100.0 \\
\hline
\end{tabular}

Table 10: Distribution of study group as per outcome of pregnancy

\begin{tabular}{ccc}
\hline Outcome & No. of Females & Percentage \\
\hline Good & 43 & 78.2 \\
Bad & 12 & 21.8 \\
\hline Total & 55 & 100.0 \\
\hline
\end{tabular}

Table 11: Distribution of study group as per IUFD

\begin{tabular}{ccc}
\hline IUFD & No. of Females & Percentage \\
\hline Yes & 7 & 12.7 \\
No & 48 & 87.3 \\
\hline Total & 55 & 100.0 \\
\hline
\end{tabular}

Table 12: Association ANA variable with other Parameters

\begin{tabular}{|c|c|c|c|c|c|c|c|}
\hline \multirow{2}{*}{$\begin{array}{c}\text { Parameters } \\
\text { HTN }\end{array}$} & \multicolumn{4}{|c|}{ ANA } & Chi square Test & \multirow[t]{2}{*}{ P-Value } & \multirow{2}{*}{$\begin{array}{c}\text { Significant at } 5 \% \\
\text { Level }\end{array}$} \\
\hline & $1+$ & $2+$ & Negative & Total & & & \\
\hline $\mathrm{PIH}$ & 14 & 6 & 1 & 21 & 0.347 & 0.841 & No \\
\hline No & 21 & 10 & 3 & 34 & & & \\
\hline Total & 35 & 16 & 4 & 55 & & & \\
\hline \multicolumn{8}{|l|}{ Diabetes MOD } \\
\hline GDM & 7 & 2 & 2 & 11 & 2.813 & 0.245 & No \\
\hline No & 28 & 14 & 2 & 44 & & & \\
\hline Total & 35 & 16 & 4 & 55 & & & \\
\hline \multicolumn{8}{|l|}{ Thyroid function test } \\
\hline Hypothyroidism & 4 & 7 & 0 & 11 & 9.383 & 0.052 & No \\
\hline Hyperthyroidism & 5 & 2 & 0 & 7 & & & \\
\hline No & 26 & 7 & 4 & 37 & & & \\
\hline Total & 35 & 16 & 4 & 55 & & & \\
\hline \multicolumn{8}{|l|}{$\mathrm{ACL}$} \\
\hline Negative & 32 & 14 & 2 & 48 & 5.548 & 0.062 & No \\
\hline Positive & 3 & 2 & 2 & 7 & & & \\
\hline Total & 35 & 16 & 4 & 55 & & & \\
\hline \multicolumn{8}{|l|}{ USG } \\
\hline Abnormal & 20 & 13 & 2 & 35 & 3.104 & 0.212 & No \\
\hline Normal & 15 & 3 & 2 & 20 & & & \\
\hline Total & 35 & 16 & 4 & 55 & & & \\
\hline \multicolumn{8}{|l|}{ Treatment taken } \\
\hline Yes & 27 & 11 & 1 & 39 & 4.782 & 0.092 & No \\
\hline No & 8 & 5 & 3 & 16 & & & \\
\hline Total & 35 & 16 & 4 & 55 & & & \\
\hline \multicolumn{8}{|l|}{ LA } \\
\hline Negative & 34 & 15 & 4 & 53 & 0.524 & 0.770 & No \\
\hline Positive & 1 & 1 & 0 & 2 & & & \\
\hline Total & 35 & 16 & 4 & 55 & & & \\
\hline
\end{tabular}


MedPulse - International Journal of Gynaecology, ISSN: 2579-0870, Online ISSN: 2636-4719, Volume 13, Issue 3, March 2020 pp 75-80

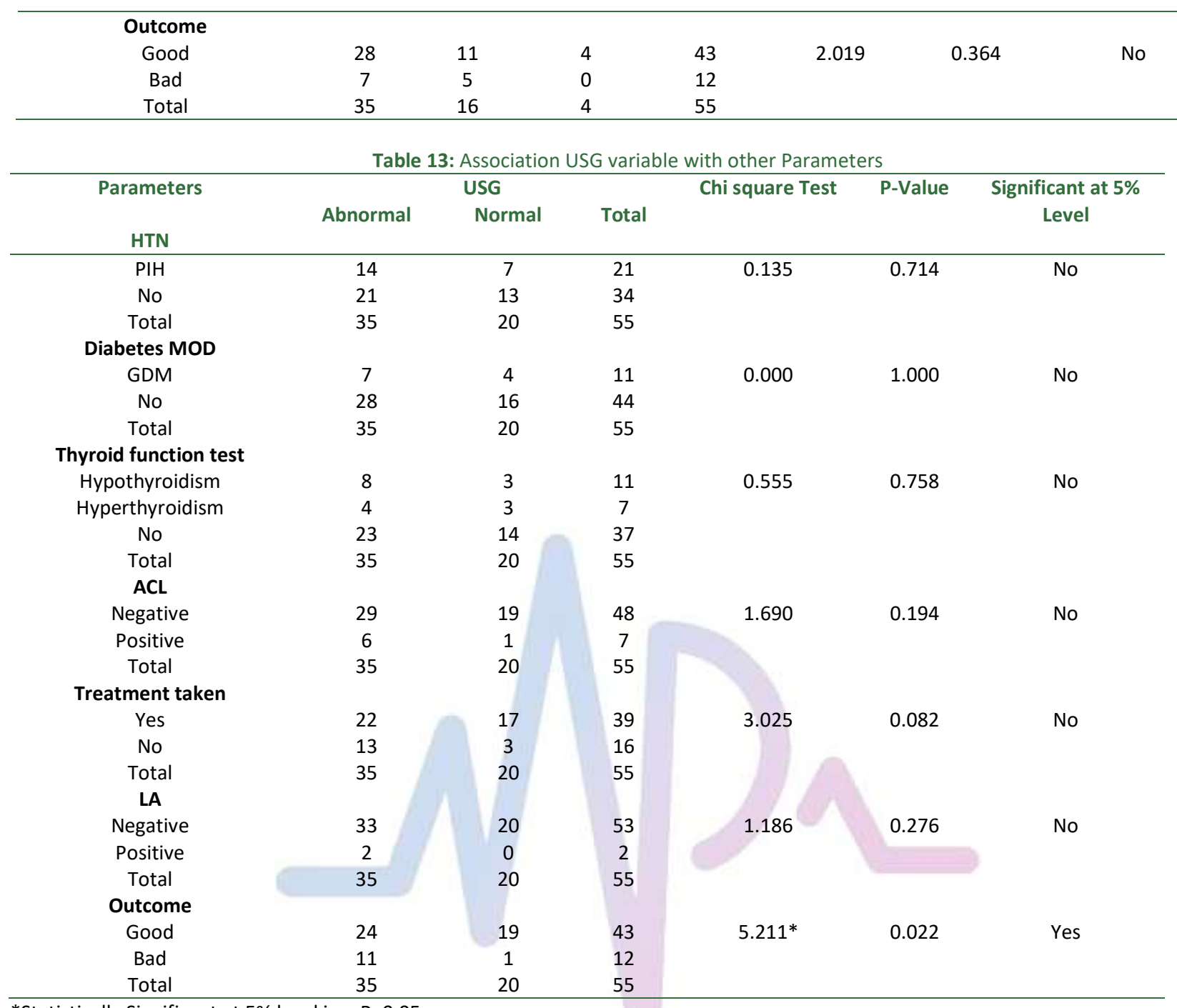

*Statistically Significant at $5 \%$ level i.e., $\mathrm{P}<0.05$.

Table 14: Association Outcome variable with other Parameters

\begin{tabular}{|c|c|c|c|c|c|c|}
\hline \multirow[t]{2}{*}{ Parameters } & \multicolumn{3}{|c|}{ Outcome } & \multirow[t]{3}{*}{ Chi square Test } & \multirow[t]{3}{*}{ P-Value } & \multirow[t]{3}{*}{ Significant at $\mathbf{5 \%}$ Level } \\
\hline & Bad & Good & Total & & & \\
\hline \multicolumn{4}{|l|}{ HTN } & & & \\
\hline $\mathrm{PIH}$ & 2 & 19 & 21 & 3.010 & 0.083 & No \\
\hline No & 10 & 24 & 34 & & & \\
\hline Total & 12 & 43 & 55 & & & \\
\hline \multicolumn{7}{|l|}{ Diabetes MOD } \\
\hline GDM & 1 & 10 & 11 & 1.306 & 0.253 & No \\
\hline No & 11 & 33 & 44 & & & \\
\hline Total & 12 & 43 & 55 & & & \\
\hline \multicolumn{7}{|l|}{ Thyroid function test } \\
\hline Hypothyroidism & 5 & 6 & 11 & 5.739 & 0.057 & No \\
\hline Hyperthyroidism & 0 & 7 & 7 & & & \\
\hline No & 7 & 30 & 37 & & & \\
\hline \multicolumn{7}{|l|}{ Total } \\
\hline \multicolumn{7}{|l|}{$\mathrm{ACL}$} \\
\hline Negative & 10 & 38 & 48 & 0.214 & 0.643 & No \\
\hline Positive & 2 & 5 & 7 & & & \\
\hline
\end{tabular}




\begin{tabular}{ccccccc} 
Total & 12 & 43 & 55 & & & \\
Treatment taken & & & & & & \\
Yes & 2 & 37 & 39 & $21.892^{*}$ & $<.001$ & \\
No & 10 & 6 & 16 & & & Nos \\
Total & 12 & 43 & 55 & & & \\
LA & 12 & 41 & 53 & 0.579 & 0.447 & \\
Negative & 0 & 2 & 2 & & & \\
Positive & 12 & 43 & 55 & & \\
Total & & & & & \\
\hline
\end{tabular}

*Statistically Significant at 5\% level i.e., $\mathrm{P}<0.05$.

In this study of 55 patients average age of patients was between 2042 years with a mean of 30 . Average of married life was between $1 \_12 \mathrm{yr}$ with a mean of $4.45 \mathrm{yr}$. $38.2 \%$ patients had $\mathrm{PIH}, 20 \%$ patients had GDM, 20\% patients had hypothyroidism, $12.7 \%$ patients had hyperthyroidism. In $63.6 \%$ patients USG findings were abnormal (IUGR, Oligohydramnios, IUFD,). ANA1+ was present in $63.6 \%$ of patients, ANA2+ was present in $29.1 \%$,ACLA in $12.7 \%$ patients and LAC in $3.3 \%$ patients. $78.2 \%$ of patients had taken treatment and $78.2 \%$ patients had good fetal outcome while $21.8 \%$ patients had bad fetal outcome and $12.7 \%$ ended up with IUFD or missed abortions. Those who were ANA $1+$ or $2+$ positive, $40 \%$ and $37.5 \%$ patients had PIH respectively with $\mathrm{p}$ value of 0.84 which is not significant statistically. $20 \%$ and $12.5 \%$ patients had GDM with ANA+ and ANA2+ respectively with $p$ value of 0.245 (not significant) $11.42 \%$ and $43.75 \%$ patients had hypothyroidism in presence of ANA+ and ANA2 + respectively and hyperthyroidism was present in $14.2 \%$ and $12.5 \%$ of patients respectively with $p$ value of 0.052 which is not significant statistically. Abnormal USG findings were present in $57.14 \%$ and $81.25 \%$ of patients with ANA+ and ANA $2+$ positive patients respectively with $\mathrm{p}$ value of 0.212 (not significant). $72.14 \%$ patients with ANA+ and $68.75 \%$ patients with ANA $2+$ had taken treatment. Bad fetal outcome was $20 \%$ and $31.25 \%$ in ANA + and ANA 2+ positive patients respectively with $\mathrm{p}$ value of 0.364 (statistically not significant). 8 patients were APLA (ACL antibodies + LA antibodies) positive, out of which $25 \%$ had PIH, $12.5 \%$ had GDM, and $25 \%$ had hypothyroidism. It was found that $63.6 \%$ patients had abnormal USG findings, $21.8 \%$ patients had bad fetal outcome and $70.9 \%$ patients had taken treatment. It was also observed in this study that multiparae ( $>1$ issue) and married life 5 or more years, had better prognosis. P values of these parameters is 0.018 and 0.024 respectively which is statistically significant. Bad fetal outcome in presence of PIH with APLA positive patients was $16.6 \%$, in GDM patients, $8.33 \%$, in hypothyroidism patients $41.66 \%$, hyperthyroidism patients $0 \%$, in patients with abnormal USG findings $31.42 \%$ and in those who had not taken treatment it was $83.33 \%$. Significant impact on pregnancy outcome was associated with abnormal USG findings and treatment taken or not. $\mathrm{P}$ value of these parameters are 0.022 and 0.001 which is statistically significant.

\section{DISCUSSION}

Association of APLA with adverse pregnancy outcome is documented since long however association of ANA with recurrent pregnancy loss or adverse course during pregnancy is still debatable. Kutteh WH et al from USA in 1996 found that $17.3 \%$ patients with recurrent pregnancy loss(RPL) had positive ACLA, $10.1 \%$ women were positive for another APLA ${ }^{5}$ while in our study it was $16 \%$.Kumar KSD et al from India in 2002 reported importance and usefulness of screening of APLA in women experiencing RPL as a mandatory routine for instituting efficient therapeutic regimen for a successful outcome of pregnancy (6), in this study successful outcome. S Velayuthaprabhu et al from India in 2005 reported that levels of aCL IgG and aPS IgG were detected as $40 \%$ and $19 \%$ respectively in women with history of recurrent abortions (7). Rosalind et al from Pittsburgh found that previous adverse pregnancy outcome was the most important risk factor for an adverse outcome in subsequent pregnancy ${ }^{8}$. Luis H. et al in 1992 reported that efficacy of treatment with prednisolone and aspirin in cases of RPL with the antiphospholipid syndrome. They found that prior to therapy, the rate of live-born babies was $15.6 \%$, and after therapy, it was $100 \%$. In this study we also got $100 \%$ successful pregnancy outcome. There was no significant adverse effects on either mother or babies $^{9}$.In May 1999 J Associate Physician ( authorized by Chakraborty S, Bhunia C, Bhattacharya DK, ) recommended that detection of APL antibodies must be considered in women with previous pregnancies complicated by unexplained fetal wastages ${ }^{10}$. Giasuddin et al from Bangladesh reported in 2010 that prevalence of ACA in patients with recurrent pregnancy loss was $37.1 \%$ while in control group it was $5.4 \%{ }^{11}$.

\section{CONCLUSION}

From this progressive case study we conclude that ANA and APLA should not be done as a routine but only in risk cases and in patients with poor pregnancy outcome in 
previous pregnancies as to improve pregnancy course as well as outcome.

\section{REFERENCES}

1. Asherson RA, Khamashta MA, Ordi-Ros J, Derksen RH, Machin SJ, Barquinero J, et al. The "primary" antiphospholipid syndrome: major clinical and serological features. Medicine (Baltimore). Nov 1989;68(6):366-74.

2. Kochenour NK, Branch DW, Rote NS, et al. A new postpartum syndrome associated with antiphospholipid antibodies. Obstet Gynecol. Mar 1987; 69(3 Pt 2):460-8.

3. Cowchock FS, Reece EA, Balaban D, et al. Repeated fetal losses associated with antiphospholipid antibodies: a collaborative randomized trial comparing prednisone with low-dose heparin treatment. Am J Obstet Gynecol. May 1992;166(5):1318-23.

4. Wilson WA, Gharavi AE, Koike T, Lockshin MD, Branch $\mathrm{DW}$, Piette JC, et al. International consensus statement on preliminary classification criteria for definite antiphospholipid syndrome: report of an international workshop. Arthritis Rheum. Jul 1999;42(7):1309-11.

5. Antiphospholipid antibodies (APA) and recurrent pregnancy loss: treating a unique APA positivepopulation* R.D. Franklin and W.H. Kutteh1,Hum.Reprod. (2002) 17 (11):2981,2985.doi: 10. 1093/humrep/17.11.298.
6. Kumar KSD, Jyothy A, Prakash MS, Rani HS, Reddy PP from Institute of Genetics and Hospital for Genetic Diseases, Osmania University, Begumpet, Hyderabad, India and National Institute of Nutrition, ICMR, Tarnaka, Hyderabad, India. Journal of Postgraduate Medicine, Vol. 48, Issue 1, 2002 .

7. Velayuthaprabhu S, Archunan G. Evaluation of anticardiolipin antibodies and antiphosphatidylserine antibodies in women with recurrent abortion. Indian J Med Sci. 2005 Aug; 59(8):347-52.

8. Rosalind Ramsey-Goldman ${ }^{1}{ }^{2}$, Joan E. Kutzer ${ }^{1}$, et all Pregnancy Outcome and Anti-Cardiolipin Antibody in Women with Systemic Lupus Erythematosus, Am. J. Epidemiol. (1993) 138 (12):1057-1069.

9. Luis H. Silveira, M.D.Chris L. Hubble, et all American Journal of Medicine Volume 93, Issue 4 , in October 1992 published a paper on Prevention of anticardiolipin antibody-related pregnancy losses with prednisone and aspirin.

10. Chakrabarti S, Bhunia C, Bhattacharya DK, The prevalence of antiphospholipid antibodies in cases of recurrent pregnancy loss, J Assoc Physicians India. 1999 May; 47(5):496-8.

11. Giasuddin AS, Mazhar I, Haq AM Prevalence of anticardiolipin antibody in Bangladeshi patients with recurrent pregnancy loss, Bangladesh Med Res Counc Bull. 2010 Apr;36(1):10-3.

\section{Source of Support: None Declared Conflict of Interest: None Declared}

